\title{
Study on Quality and Safety Risk Factors of Edible Fungi and Preventive Measures: Take China as an Example
}

\author{
Fengjuan Jia, Yueming Wang, Zhiqing Gong, Wenjia Cui, Yansheng Wang, Wenliang Wang* \\ Institute of Agro-Food Science and Technology, Shandong Academy of Agricultural Sciences, Jinan, China \\ Email: jfj.5566@163.com, wangym1969@sohu.com, 147372843@qq.com,165703519@qq.com,296138032@qq.com, *cywwl@163.com
}

How to cite this paper: Jia, F.J., Wang, Y.M., Gong, Z.Q., Cui, W.J., Wang, Y.S. and Wang, W.L. (2016) Study on Quality and Safety Risk Factors of Edible Fungi and Preventive Measures: Take China as an Example. Open Access Library Journal, 3: e3154.

http://dx.doi.org/10.4236/oalib.1103154

Received: October 18, 2016

Accepted: November 10, 2016

Published: November 14, 2016

Copyright $\odot 2016$ by authors and Open Access Library Inc.

This work is licensed under the Creative Commons Attribution International License (CC BY 4.0).

http://creativecommons.org/licenses/by/4.0/

\begin{abstract}
Agricultural product quality and safety are local and international research hotspots. China was the world's largest producer of edible fungi, but quality and safety problems restricted the sustainable development of this industry, which is the sixth largest class of agricultural products in China. Consumers have become increasingly concerned with the quality and safety of edible fungi because they can be easily contaminated with heavy metals, chemical pesticide residues, chemical additives, and microbes. This study summarized the safety problems of edible fungi to develop control measures for ensuring their quality and safety.
\end{abstract}

\section{Subject Areas}

Agricultural Science, Food Science \& Technology

\section{Keywords}

Edible Fungi, Quality and Safety, Heavy Metal, Pesticide Residue,

Chemical Agents, Microbial Pollution Problems

\section{Introduction}

As a health food, edible fungi are consumed by humans worldwide because they are low in calories and high in vegetable proteins, vitamins, iron, chitin, fiber, and minerals. Edible fungi are becoming increasingly important in our diet owing to their nutritional, organoleptic, and pharmacological characteristics [1] [2]. Over the past few years, considering the sustained growth of demand and production of edible fungi, China has become the world's largest cultivator, consumer, and exporter of edible fungi [3]. In 2003, the annual output of edible fungi of all kinds in China totaled 3148 tons, and the 
market value was over 207.6 billion CNY, with over 2 million people employed in edible fungus industry [Sources of cited figures: Edited data from China Edible Fungi Association website (http://www.cefa.org.cn/) and China Edible Fungus Business website (http://www.mushroommarket.net/)]. According to statistics from China Industry Information and China Edible Fungi Association's websites, China's edible fungus consumption structure in 2013 is showed in Figure 1 [4]. Thus, scientists predicted that edible fungi will become one of the major functional foods in the 21st century. Edible fungi, serving as one of China's agricultural developments among "three agriculture" and "white agriculture," played a more important role in agricultural production. However, the data of national routine monitoring of the agricultural product quality and safety from the Chinese Ministry of Agriculture showed that pesticide residues, as well as poisonous and harmful substances were detected in the industry of edible fungi. With the continuous improvement of living standards, the awareness of environmental protection and health consciousness gradually enhanced. Edible fungi served as one of people's favorite food dishes, but the quality and safety problem attracted increasing consumer attention. To date, how to effectively control the quality safety of edible fungi, how to deal with the local and international market demand for edible fungus products, and how to ensure the sustainable development of edible fungus industry have become urgent problems. Therefore, excess levels of heavy metals, pesticide residues, and chemical agents, as well as microbial pollution problems of edible fungi were analyzed in the present study. Corresponding prevention control measures were proposed, providing a theoretical basis to solve the problems in the quality and safety of edible fungi.

\section{Quality and Safety Problems of Edible Fungi}

\subsection{Heavy Metal Pollution}

Heavy metals are elements with density greater than $4.5 \mathrm{~g} / \mathrm{cm}^{3}$, including copper, lead,

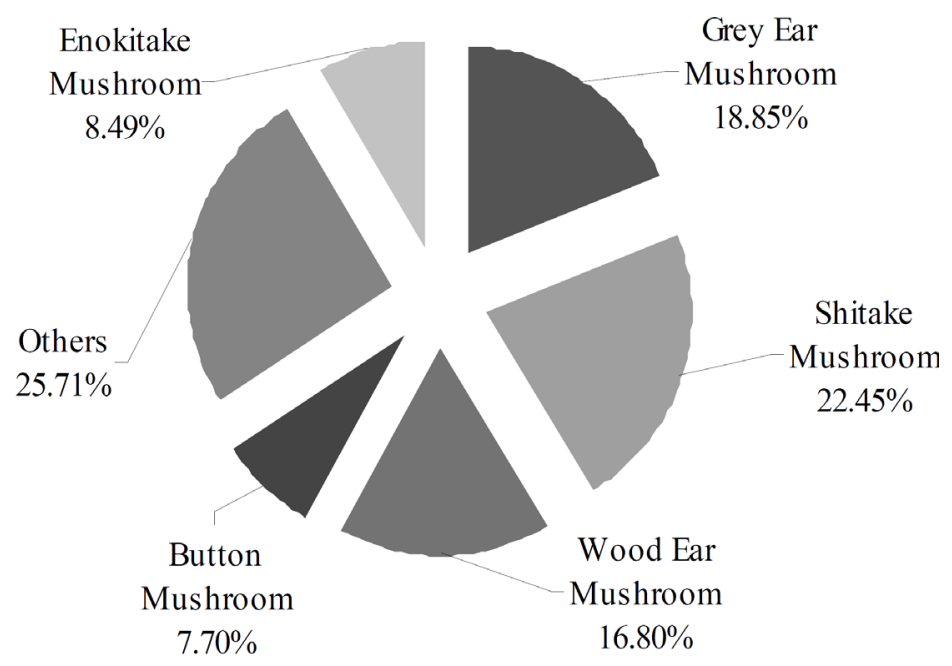

Figure 1. Mushroom consumption in China in the year 2013 [4]. 
zinc, iron, cobalt, nickel, manganese, and dozens of other of trace elements, which are either beneficial to the human body or harmful for human health. Edible fungi demonstrate the ability of bioaccumulation and transformation in heavy metals, thereby enriching people with beneficial trace elements but simultaneously absorbing some harmful trace elements. Heavy metals are not easy to be depredated by microorganisms, but can be easily enriched in biological bodies. Heavy metals enter the human body through the food chain and cause some diseases, such as kidney and liver organ lesions, which are harmful to human health. The source of heavy metals in edible fungi is from its growth-required substrate, water, soil, air, and other factors. Generally, the growth cycle of edible fungi is short. Thus, the absorption amount of heavy metals through the edible fungus body surface is limited. The most possible way of adsorption of heavy metals by edible fungi is through the fungus hypha from the cultivation matrix. Wild edible fungi adsorb heavy metals from growth soil, organic matter, water, and air pollution, whereas heavy metals of the artificially cultivated edible fungi are often adsorbed from the cultivation matrix, soil, air, and water. In nearly 5 years, the data from the Chinese Ministry of Agriculture of the special monitoring and risk assessment of heavy metals in edible fungi showed that the qualification rate of heavy metals in edible fungi was over 98\%, entailing a risk for quality and safety. In China, the "Edible Health Standard," "Pollution-free Edible Fungus," "Green Edible Fungi," and other national and industrial standards for heavy metal content in edible fungi have proposed that all edible fungi with excessive heavy metal pollution should not be consumed [5].

\subsection{Excessive Chemical Pesticide Residue}

According to function, chemical pesticides can be classified into pesticides, fungicides, herbicides, and plant-growth regulators. According to toxicity, chemical pesticides can be classified into highly toxicity, high-toxicity, and low-toxicity pesticides. Pesticide residue pollution of edible fungi mainly results from either the cultivation matrix indirectly or chemical pesticide direct pollution in control of diseases and pests. Majority of the edible fungus cultivation matrix comes from the cutoff of agriculture and forestry products. However, in the process of agricultural production, pesticides are generally used to control insects, prevent diseases, and eliminate weeds. Considering the high residue and bioaccumulation features of chemical pesticides, the extensive use of these pesticides brought pesticide residue pollution in agriculture and forestry products, as well as pollution of the ecological environment, causing accumulation of pesticide residues in the edible fungus culture medium. The residues of pesticides in edible fungi can also be accumulated and detained in the body for a long time, causing chronic poisoning and harming people's health.

\subsection{Chemical Pollution}

\subsubsection{Fluorescent Whitening Agent}

The fluorescent whitening agent is a kind of chemical dye that can absorb visible light or ultraviolet light and reflect blue white phosphorus light, which can be dealt with to 
improve the whiteness and luster of objects. In China, the fluorescent whitening agent is mainly used in papermaking, synthetic detergents, plastics, and textile industry. Ingestion of the fluorescent whitening agent can cause cell mutation, toxic accumulation in the liver and other vital organs, or even cancer. In China, the Hygienic Standards of Food Additives stipulate that the fluorescent whitening agent is strictly prohibited from being used in the food industry. On the one hand, the fluorescent whitening agent pollution of edible fungus comes from packaging materials, which were processed by this substance. On the other hand, soaking edible fungi with the fluorescent whitening agent or adding this substance to food by peddlers can also lead to the fluorescent whitening agent pollution.

\subsubsection{Sulfur Dioxide Pollution}

Sulfur dioxide is a colorless and nonflammable gas with the abilities of strong reducibility, whitening, and refreshment. Excessive intake of sulfur dioxide for a long time can cause bowel dysfunction. According to the China Ministry of Public Health, the content of sulfur dioxide should be not higher than $0.05 \mathrm{~g} / \mathrm{kg}$ as a food additive. However, in the production and processing of edible fungi, some unscrupulous traders often illegally use excessive sulfur dioxide to fumigate edible fungi in order to achieve the purpose of whitening and keeping fresh of edible fungi, leading to excess sulfur dioxide in edible fungi. Additionally, illegal addition of sulfur dioxide is another source of the edible fungus additive pollution.

\subsection{Microbial Pollution}

Microorganisms, such as bacteria, fungi, viruses, and actinomycetes, distribute extensively in nature. When the growth condition is suitable, these microorganisms will be increased and reproduced massively. After contaminating edible fungi, microorganisms decompose the edible fungus sugar, protein, and fat, as well as other nutrients, by using their various enzymes, which can destroy the edible fungus sensory properties, reduce the nutritional values, and even corrupt the fruiting bodies, eventually leading to deterioration. Microorganism pollution of edible fungi mainly involves two types of microbes, namely, bacteria and fungi. The common fungal categories are Aspergillus, Penicillium, Trichoderma viride, sickle bacteria genera, cross-linking spore mildew, pink single-ended spore mildew, and black grape grain mildew [6] [7]. They secrete toxin to inhibit the human body DNA replication and protein synthesis, reduce the immune response, and cause serious harm to human body health. Acute poisoning often occurs when substantial amount of edible fungi is ingested with toxin one time, and for a long time, intake of edible fungi with a small amount of toxin can cause chronic poisoning or even cancer [7].

\section{Factors Adversely Affecting the Quality and Safety of Edible Fungi}

Various factors affect the quality and safety of edible fungi. In addition to the weak supervision and incomplete standard, most of the factors are established in the produc- 
tion and processing of edible fungi, including heavy metal pollution, the use of cultivation matrix with pesticide residue, improper cultivation environment pollution, pest control, illegal use of chemical reagents, and undemanding health measures.

\subsection{Cultivation Matrix Leads to Heavy Metal Pollution and Pesticide Residues}

The raw material of edible fungus cultivation matrix is extensive, mainly including the processing residues of sawdust, cotton seed shell, corn cob, straw, bran, and other green plants. The auxiliary material is usually $\mathrm{Ca}, \mathrm{Mg}$, $\mathrm{P}$, or other mineral fertilizers. Considering the bio-enrichment of heavy metals by green plants, when the soil is polluted by heavy metals, the heavy metal content of edible fungus cultivation matrix is consequently high. Additionally, the cadmium content is higher in phosphate rock, and part of cadmium enters into phosphate fertilizer production. Thus, with the use of phosphate fertilizer, cadmium simultaneously enters the cultivation matrix. Edible fungi grow in the cultivation matrix with high heavy metal content, and edible fungus hypha absorb and transfer the heavy metals to the fruiting body. With the enrichment effect, the heavy metal content in the fruiting body is much higher than normal levels [8]. Research showed that when cultivation matrix is seriously polluted, the largest absorption amount of lead in Lentinus edodes, Auricularia auricula, and Pleurotus ostreatus can reach $150 \mathrm{mg} / \mathrm{kg}$ to $200 \mathrm{mg} / \mathrm{kg}$. The amount of lead absorption by Agaricus bisporus can be up to $30 \mathrm{mg} / \mathrm{kg}$. The cumulative effect of $L$. edodes on Cd is more outstanding, and the largest cumulant can reach $180 \mathrm{mg} / \mathrm{kg}$ [9]. In the process of agricultural production, considering the heavy use of chemical pesticides, the pesticide residues in the edible fungus cultivation raw material are high in amount. Subsequently, the toxic and harmful substances enter into edible fungi, causing excessive pesticide residues.

\subsection{Prevention and Control of Diseases and Insect Pests Produce Pesticide Residues}

According to the Law of Pesticide Management in the People's Republic of China, toxic and highly toxic pesticides may not be used in vegetable production. This ordinance should be completely applied to the edible fungus industry. However, in the edible fungus production process, toxic and highly toxic pesticides are often used for the prevention and control of plant diseases and insect pests for various reasons. Pesticide residues produce toxic effects, causing problems in edible fungus quality and safety. Although low-toxicity insecticides and fungicides are allowed to be used in edible fungus production, the country follows strict rules regarding the use of these pesticides, and blindly using or misusing of pesticides easily produces the poisonous material residues. According to the "Pollution-free Food Safety Technical Requirements of Edible Fungus Cultivation Matrix," plant-growth regulators are not allowed to be used in the edible fungus cultivation matrix [5]. However, under the drive of economic interests, some edible fungus production enterprises often add plant-growth regulators to the cultivation matrix to obtain higher output and income, which brought unexpected negative effects on the quality and safety of edible fungi. 


\subsection{Environmental Pollution Leads to Poisonous Residues and Harmful Materials}

The emission of industrial waste and the great usage of chemical pesticides pollute the growth environment of edible fungi at different levels, such as soil, atmosphere, and water, either directly or indirectly causing excessive poisonous and high harmful substance content of edible fungi. Soil-cover culture is one of the high-yield cultivation ways of edible fungi. The hyphae of edible fungi can easily absorb heavy metals from heavy-metal-polluted soil, thereby exceeding the standard of heavy metal content in edible fungi [10] [11]. Sulfur dioxide emission is one of the main atmospheric pollutants. Given the high moisture and strong water imbibition of the edible fungus fruiting body, sulfur dioxide in the air is extremely easy to be absorbed by the fruiting body. Sulfur dioxide interacts with water from the intercellular space fast to generate sulfite and bisulfite, causing the sulfite to be out of limits [12]. Additionally, the growth of edible fungi requires much water. If the contaminated water is stirred into the edible fungus cultivation matrix or sprayed fruiting body in the mushroom management period, the highly toxic and harmful substances in the fruit body will remain.

\subsection{Chemical Pollution in Edible Fungus Processing}

In edible fungus processing process, the illegal usage of chemical agents and food additives make edible fungus remain toxic or harmful substances, influencing the safety of the quality of edible fungus. The fluorescent whitening agent is an organic compound that can improve the whiteness of a material and burnish, but it is strictly prohibited for use in food processing. However, some unscrupulous traders use the fluorescent whitening agent to treat $A$. bisporus, Coprinus comatus, and other edible fungi, making the surface smooth and white in order to improve the quality of product appearance. This aggravated misconduct causes serious damage to people's health. Moreover, sodium metabisulfite or sodium sulfite solution is often used to prevent enzymatic browning of edible fungi after harvest and may cause excess sulfur content remaining in the edible fungi if the solution concentration and soak time are not strictly controlled. Fresh edible fungus, which tastes delicious and is crispy and tender, has become increasingly popular among many people. The flavor and nutritional value of some mushrooms will be significantly reduced upon processing. Thus, people often use fresh-keeping means to keep the flavor and nutritional value of these mushrooms, ensuring the supply of fresh market. Treatment with chemicals and plant-growth regulators is one of the edible fungus preservation technologies, and the chemicals and plant-growth regulators commonly include citric acid, sodium metabisulfite, sodium chloride, ascorbic acid, and N-dimethylaminosuccinimide [12]. These substances can inhibit mushroom discoloration, open umbrella, and corruption, and the application concentration of these drugs commonly ranges from $0.01 \%$ to $0.1 \%$, with some even few parts per million. Toxic and harmful substance pollution will occur if these drugs are not used in accordance with the provisions. 


\subsection{Microbial Contamination in Edible Fungus Storage and Processing}

Edible fungi sold in the market can be classified into fresh products, dry products, and deep-processing products of edible fungi. The dry and deep-processing edible fungus products are easily polluted by microorganisms during processing, transportation, storage, and sale process, leading to edible fungus deterioration. The three main pathways of edible fungus pollution are (1) pollution of raw material (fresh mushroom); (2) pollution in the processing process; and (3) pollution in the product transportation, storage, and distribution [7]. In the edible fungus cultivation process, high-pressure steam sterilization or atmospheric-pressure steam sterilization is usually adopted to sterilize the cultivation matrix. Moreover, other cultivation technologies are very strict with regard to disinfection measures. Thus, the raw fresh mushroom material probably presents low microbial contamination. The microbial contamination of edible fungi mainly occurs in during the processing, transportation, and storage process if the operation is not strictly in accordance with the requirements for health, which will lead to microbial contamination and affect the quality and safety of edible fungi.

\section{Preventive Measures to Solve the Problems}

To solve the problems of edible fungus quality and safety is a long-term and arduous task. Preventive measures include reducing the heavy metal content, reducing and eliminating the pesticides and chemicals residues, and preventing microbial contamination. Additionally, we should strengthen market supervision, as well as polish and implement the standard of quality and safety of edible fungus. The green planting and environmentally friendly processing methods should also be applied in accordance with the requirements of edible fungus standardized production to promote the improvement of product quality and safety.

\subsection{Scientific Selection of Cultivation Matrix}

The cultivation matrix selection is an important part of the edible fungus green planting. Thus, the selection cultivation matrix should be in accordance with the requirements of the edible fungus standardization production. Artificial edible fungus cultivation matrix is usually made of scraps from agriculture and forestry. To ensure the quality and safety of edible fungi, the cultivating matrix must be fresh, dry, and clean, with no mildew, no insects, and no peculiar smell. Soaking and fermentation can reduce the content of pesticide residues if sawdust is used as the main raw material of the cultivation matrix. With dung grass as the main raw material, pesticide residues and heavy metals should be tested in advance, and dung grass with low harmful content can be used as the cultivation matrix raw material. If straw and cotton seed hull are used as the main raw materials, the original crops need to be requested without high-toxic pesticide application before the harvest time for 1 month. Heavy metal and pesticide residue pollution of edible fungi should be effectively prevented, unless the producer strictly selected the raw materials for the cultivation matrix. 


\subsection{Scientific Prevention and Control of Plant Diseases and Insect Pests}

Edible fungal disease and insect prevention should insist the principle that physical and biological control comes first, and chemical control is the necessary complement to prevent and cure the diseases and insects. Combination of environmental control, cultivation, ecological, physical, and chemical measures for prevention and treatment, such as selecting excellent resistant varieties, purifying health strains, and preparing high yield substrates, should also adopt suitable cultivation and standardized management technologies for adjusting and controlling various factors in mushroom shed [13]. On the use of pesticides, the producers should follow the principle that pesticides must be used safely and strictly enforce the pesticide safety standards and rules of rational use of pesticides. Poisonous, high-toxicity, and high-residual chemical pesticides are prohibited, but they are preferred to select the microbial source and botanical pesticide formulations [14] [15]. Selecting the scientific method and controlling the pesticide usage amount and duration are sufficient to create beneficial growth environment conditions for edible fungi and to inhibit plant diseases and insect pests.

\subsection{Scientific Selection of Production Site}

Considering the significant influence of growth environment on the quality and safety of edible fungi, production areas should be located in clean and tidy places, where toxic substances in the atmosphere, water, soil, and other environments should not be excessive. Around the production areas, no industrial wastewater, waste, emissions, livestock farms, dump, and all kinds of pollution sources (such as wastewater, cement, and lime), should exist. The production site should also be far away from hospitals, schools, residential areas, and other public places to prevent mutual influence. Some disinfection measures, such as turning up the soil, solarization, and irrigation, can replace the usage of pesticides.

\subsection{Safe usage of Chemical Pesticides}

The safe usage of chemical pesticides is a powerful guarantee to avoid edible fungus toxic and harmful substance pollution during processing. The Hygienic Standards of Using Food Additives in China (GB2760) provided the definition, categories, usable range, and principles of food additives [5]. In the edible fungus processing process, the producers should strictly accord with the standards and scientifically use food additives. In addition, in edible fungus freshness preservation processing, the usage of chemicals and plant-growth regulators should be accorded to the relevant provisions.

\subsection{Prevention and Control of Microbial Contamination}

The International Food Hygiene Operation and Basic Management Law must be followed to prevent and control the edible fungus product far from microbial contamination and deterioration in the process of edible fungus processing and storage. The five aspects of prevention and control measures, namely, raw materials, processing process, technological process, three libraries (raw-material library, auxiliary material library, 
and finished-product library), and personnel health, should carried out to ensure the health requirements of edible fungus product and meet the requirement of the Health Standard for Edible Fungus (GB7096-2003) [5].

\subsection{Strengthened Supervision on the Edible Fungus Quality and Safety}

Producers and sellers should establish edible fungus quality safety records, strictly control the edible fungus base out and market access system, and carry out edible fungus self-check work earnestly. Relevant regulatory departments should intensify supervision at all levels and actively carry out routine monitoring, risk assessment, and sole rectification [16]. The quality supervisions investigate and take care of pesticides and additives illegally used in edible fungus production and circulation process, thereby completely eradicating unqualified edible fungi to market [17].

\section{Conclusion}

An increasing number of people are becoming fond of edible fungi because of the nutritional and health benefits, as well as delicious taste, but the quality and safety problems of edible fungi attract much attention. China was the world largest edible fungus production country, but the quality safety problem restricted the sustainable development of industry. This study summarized the edible fungus quality and safety problems, which cause a direct or potential harm to consumer health. At present, pesticide residue, heavy metal and microbial contamination became the main risk factors affecting the quality and safety of edible fungi. In order to ensure the healthy and sustainable development of mushroom industry, strengthening the production of the main training, enhancing the quality and safety awareness, improving the safety technical standards, vigorously promoting the standardization of production, strengthening the construction of monitoring team and improving the security service system to control the risk factor were put forward. Producers and administrators should take scientific and effective prevention and control measures by controlling every link of edible fungus production as effective means to ensure edible fungus quality and safety to meet the demand of local and international markets, guaranteeing the health and sustainable development of the edible fungus industry.

\section{References}

[1] Barros, L., Baptista, P., Correia, D., Casal, S., Oliveira, B. and Ferreira, I. (2007) Fatty Acid and Sugar Compositions, and Nutritional Value of Five Wild Edible Mushrooms from Northeast Portugal. Food Chemistry, 105, 140-145.

https:/doi.org/10.1016/j.foodchem.2007.03.052

[2] Ribeiro, B., Andrade, P., Silva, B., Baptista, P., Seabra, R. and Valentao, P. (2008) Comparative Study on Free Amino Acid Composition of Wild Edible Mushroom Species. Journal of Agricultural and Food Chemistry, 56, 10973-10979. https:/doi.org/10.1021/jf802076p

[3] Ge, Y.X. and Guo, Z.J. (2013) Comparison and Selection of Organization Modes in Edible Fungus Industry of Shandong Province. Asian Agricultural Research, 5, 33-37.

[4] Li, M. and Hu, J.L. (2014) Study on Survival Strategies of Farmers Engage in Small-Scale 
Household Cultivation of Edible Mushrooms: Take Shandong Province as an Example. Modern Economy, 5, 1092-1100. https:/doi.org/10.4236/me.2014.512100

[5] (2006) Quality Supervision, Inspection and Test Center of Microbial Fertilizer and Edible Fungus Strains in Ministry of Agriculture. The Edible Fungus Technology Standard Assembly. China Standard Publishing House, Beijing.

[6] Shen, P. (2000) Microbiology. Higher Education Press, Beijing.

[7] Jia, Y.M. (2007) Food Microbiology. China Light Industry Press, Beijing.

[8] Xu, L.H., Chen, Q.B. and Ye, C.W. (2005) The Study on the Absorption and Enrichment Regularity of Harmful Heavy Metal by Edible Fungi. Agricultural Journal of Environmental Science, 24, 42-47.

[9] Lei, J.F. and Yang, D.F. (1990) The Heavy Metal Content of Edible Fungi and the Research of Edible Fungus Heavy Metal Enrichment. Chinese Edible Fungus, 9, 14-17.

[10] Sun, M.H., Wu, X.Q. and Wei, H.L. (2007) The Control Technology Research Progress of Edible Fungi Poisonous and Harmful Substances. Chinese Lin Vice Speciality, 15, 74-77.

[11] Huang, C.Y. and Zhang, J.X. (2004) Studies on the Enrichment of Heavy Metals in Edible Fungi. Chinese Edible Fungi, 23, 7-9.

[12] Lv, Z.Z. (2006) Edible Fungi Culture. Higher Education Press, Beijing.

[13] Wu, X.Q., Huang, Z.L. and Wei, H.L. (2003) Mushrooms Pollution-Free Production Technology. China Agriculture Press, Beijing.

[14] Zhang, Z.H. (2007) Reasonable Use of Pesticides and the Standard of Pesticides Residue Limits. Chemical Industry Press, Beijing.

[15] Men, D.Y., Tan, Q. and Wang, N. (2004) The Principle of Pesticide Use in the Production of Edible Fungi. Journal of Edible Fungi, 11, 51-56.

[16] Bosona, T.G. and Gebresenbet, G. (2011) Cluster Building and Logistics Network Integration of Local Food Supply Chain. Biosystems Engineering, 4, 294-302.

[17] Aruoma, O.I. (2006) The Impact of Food Regulation on the Food Supply Chain. Toxicolo$g y, 221,119-127$. https:/doi.org/10.1016/j.tox.2005.12.024

Submit or recommend next manuscript to OALib Journal and we will provide best service for you:

- Publication frequency: Monthly

- 9 subject areas of science, technology and medicine

- Fair and rigorous peer-review system

- Fast publication process

- Article promotion in various social networking sites (LinkedIn, Facebook, Twitter, etc.)

- Maximum dissemination of your research work

Submit Your Paper Online: Click Here to Submit

Or Contact service@oalib.com 